\title{
Peculiarities of local blood microcirculation in patients with psoriasis
}

Viktor V. Dremin, Maria A. Filina, Elena V. Zharkikh, Igor O. Kozlov, Evgeny A. Zherebtsov, et al.

Viktor V. Dremin, Maria A. Filina, Elena V. Zharkikh, Igor O. Kozlov, Evgeny A. Zherebtsov, Elena V. Potapova, Nadezda S. Malaya, Natalia Y. Yakushkina, Irina A. Snimshchikova, Andrey V. Dunaev, "Peculiarities of local blood microcirculation in patients with psoriasis," Proc. SPIE 10685, Biophotonics: Photonic Solutions for Better Health Care VI, 1068532 (17 May 2018); doi: $10.1117 / 12.2305973$ 


\title{
Peculiarities of Local Blood Microcirculation in Patients with Psoriasis
}

\author{
Viktor V. Dremin*,a, Maria A. Filina ${ }^{\mathrm{a}}$, Elena V. Zharkikha, Igor O. Kozlova, \\ Evgeny A. Zherebtsov ${ }^{b}$, Elena V. Potapova ${ }^{a}$, Nadezhda S. Malaya ${ }^{a, c}$, \\ Natalia Y. Yakushkina ${ }^{c}$, Irina A. Snimshchikova ${ }^{a}$, Andrey V. Dunaev ${ }^{a}$ \\ ${ }^{a}$ Orel State University named after I.S. Turgenev, 95 Komsomolskaya St, Orel, 302026, Russia; \\ ${ }^{\mathrm{b}}$ Aston Institute of Photonic Technologies, Aston University, Aston Triangle, Birmingham, B4 7ET, UK; \\ ${ }^{c}$ Orel Regional Dermatovenereological Dispensary, 68 Pushkina St, Orel, 302030, Russia
}

\begin{abstract}
Local hemodynamic parameters were studied by means of laser Doppler flowmetry in 15 patients with psoriasis in the stationary stage, who have plaques on the inner surface of the forearm.

LDF signals recorded at the site of psoriatic lesions of the tissue as well as in the intact tissue at a distance of $1-2 \mathrm{~cm}$ from the affected area were analysed. LDF signals were postprocessed by continuous wavelet transform using the Morlet wavelet.
\end{abstract}

Keywords: laser Doppler flowmetry, blood microcirculation, blood flow oscillations, psoriasis.

\section{INTRODUCTION}

Psoriasis is a systemic inflammatory disease that provokes an increase in the release of anti-inflammatory cytokines from immune cells and the chronic activation of the congenital and adaptive immune system. This leads to systemic damage to internal organs and development of pronounced cosmetic defects. According to statistics, psoriasis is one of the most common chronic recurrent dermatoses, occurs in $2-3 \%$ of the world's population ${ }^{1}$ and is associated with numerous concomitant diseases, including rheumatological and cardiovascular complications. Ordinary psoriasis is the most common form of psoriasis. It is observed in $80-90 \%$ of all patients with psoriasis. This type of psoriasis is most often manifested in the form of inflamed areas, covered with gray or silvery-white, easily peeling, scaly, dry and thickened skin.

In the pathogenesis of psoriasis, an important distinctive feature is microcirculatory disorders that occur before the clinical manifestations of the disease. With psoriasis in a stationary stage of the disease, the capillaries are elongated, thickened and expanded ${ }^{2,3}$.Despite a significant amount of evidence demonstrating abnormal blood microcirculation in psoriasis it is still not entirely clear when these microcirculatory changes appear in the skin of psoriasis patients and what are the causes of these disorders.

In this work, it is proposed to use the laser Doppler flowmetry (LDF) method to study microcirculation intensity, as well as wavelet analysis to estimate microcirculation oscillations. This technology is successfully used to study the blood microcirculation in people with rheumatic diseases ${ }^{4-6}$, diabetes mellitus ${ }^{7,8}$, etc.

The aim of this work is to experimentally study and analyse the possibilities of the laser Doppler flowmetry method and the spectral analysis of the obtained data for the diagnosis of microcirculatory disorders in the tissues of psoriatic plaques and the clinically uninjured skin of the upper limbs of patients with psoriasis.

*dremin_viktor@mail.ru; phone+7 (953) 612-77-13; bmecenter.ru/en 


\section{MATERIAL AND METHODS}

In experimental studies 15 patients (11 men and 4 women, $47 \pm 15$ years, Orel Regional Dermatovenereological Dispensary, Orel, Russia) participated. All patients had a diagnosis of plaque psoriasis of the stationary stage. The study included patients who had plaques on the inner surface of the forearm (Figure 1).

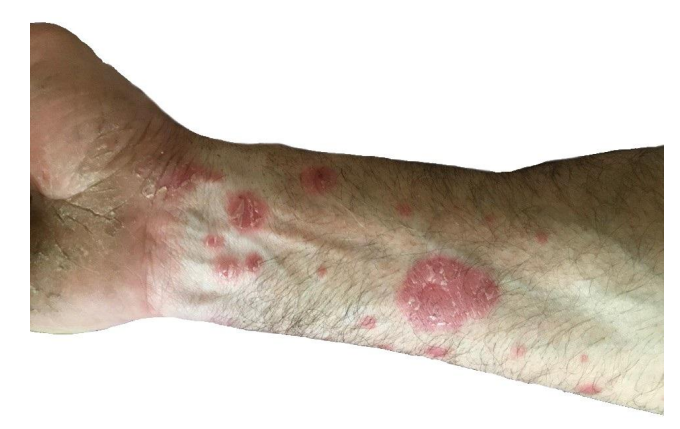

Figure 1. Psoriatic lesions on the inner surface of the forearm.

The study did not include volunteers with cardiovascular, bronchopulmonary or neuroendocrine system disease comorbidities, nor with diseases of the gastrointestinal tract, liver, kidneys, blood and any other serious chronic diseases, which could have an impact on the diagnostic results. Volunteers with a history of alcoholism, drug addiction and drug abuse were also excluded.

The study protocol was approved at a meeting of the ethics committee at Orel State University named after I.S. Turgenev from 16/11/2017 (minutes of the meeting №10). After receiving the description of the protocol, all participating volunteers signed an informed consent form.

The measurements were made on the skin of the inner side of the forearm: in the center of the psoriatic plaque and $1-2 \mathrm{~cm}$ from it (intact tissue). The studies were conducted in conditions of physical and mental rest of the patient 2 hours after eating, with adaptation to the room temperature of $20-23^{\circ} \mathrm{C}$ in the sitting position. The studies were performed using the LDF channel of the multifunctional laser non-invasive diagnostic system LAKK-M (SPE "LAZMA" Ltd., Russia) with a wavelength of the probing radiation of $1064 \mathrm{~nm}^{9,10}$. The Morlet wavelet transform was used for the frequency analysis of registered signals ${ }^{11}$.

During the experimental studies, the microcirculation index was recorded for $10 \mathrm{~min}$ at the center of the psoriatic lesion of the tissue, and $10 \mathrm{~min}$ on the intact skin at a distance of $1-2 \mathrm{~cm}$ from the affected area. The total duration of one experimental study was 20 minutes. The time-averaged amplitude of the blood flow oscillations was estimated from the maximum values in the corresponding frequency range - endothelial, neural, myogenic, respiratory and cardiac ${ }^{9}$.

A Mann-Whitney $U$-test test was used to identify differences between the two groups. Statistically significant was the probability $p<0.01$.

\section{EXPERIMENTAL RESULTS AND DISCUSSION}

When analyzing the experimental data, it was found that the hemodynamic parameters of the microcirculatory bed in the center of psoriatic plaques differ from the same parameters measured in the skin, uninvolved in the pathological process.

The Figure 2a shows the averaged recorded LDF signals in the center of the psoriatic plaque (red line) and at a distance of 1-2 cm from the lesion (blue line). The standard deviation values are shown in transparent color. An example of wavelet analysis of LDF signals is shown in Figure $2 b$.

As can be seen from Figures 2a and 3, in the zone of the psoriatic plaque, an increase in the average perfusion and a significant dominance of all types of oscillations are observed in comparison with the area of intact skin. 


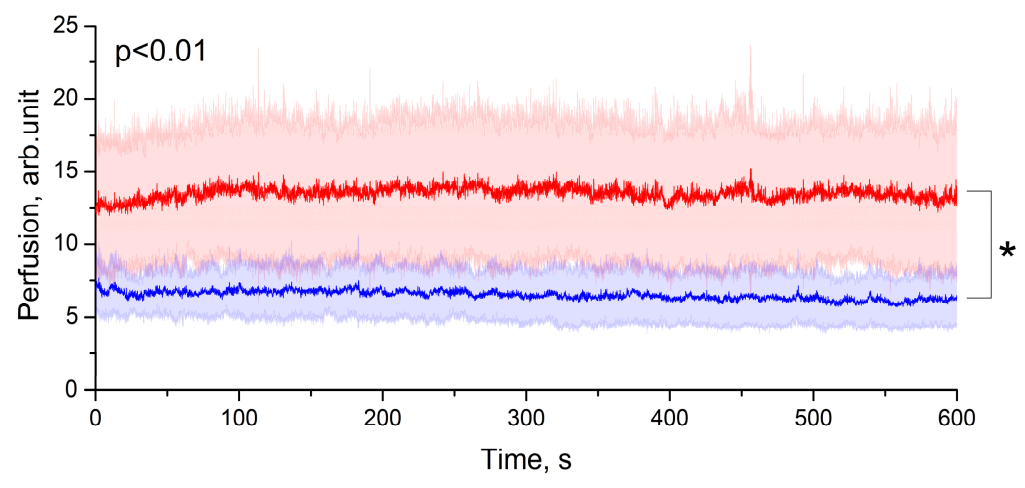

(a)

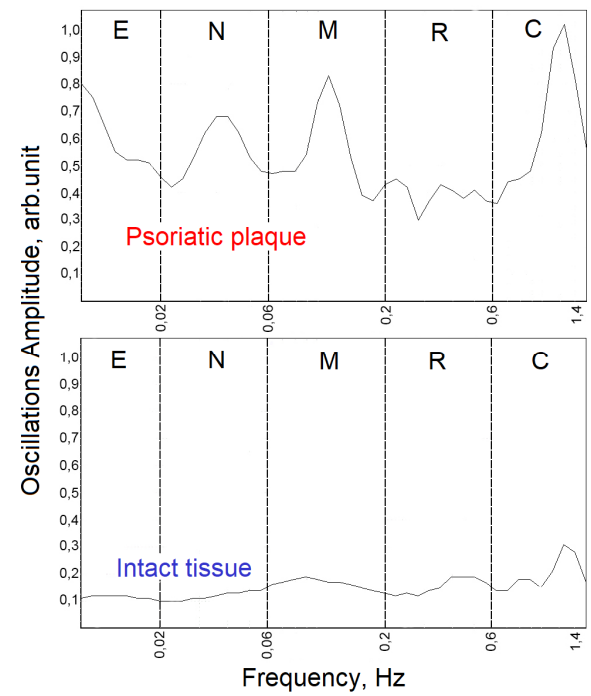

(b)

Figure 2. Averaged LDF signals in the center of the psoriatic plaque (red line) and at a distance of 1-2 $\mathrm{cm}$ from the lesion (blue line) (a) and a typical example of wavelet analysis of LDF signals (b).

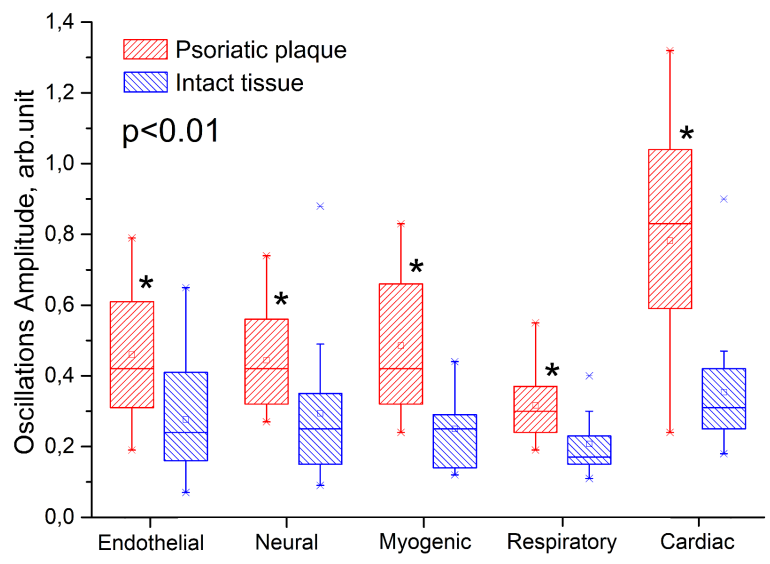

Figure 3. Comparison of amplitudes of blood flow oscillations in the zone of psoriatic lesion (red shading) and intact tissue (blue shading). In each box, the central line is the median of the group, while the edges are the 25 th and 75 th percentiles.

These results are correlated with the data obtained earlier in studies of blood flow in psoriatic lesions ${ }^{12,13}$. Vascular anomalies in psoriatic plaques extend to the deep vessels (terminal arteries and arterioles of the reticular dermis). Hyperplastic epidermis regulates these vascular changes in order to support increased nutritional requirements during disease activity ${ }^{13}$. An increase in the level of metabolism in the focus of inflammation causes an active influx of blood into the capillary bed.

It should be noted that the increase in the level of the LDF signal can occur due to an increase in the probing depth, which is based on optical effects ${ }^{14}$. Psoriasis is accompanied by acanthosis. There is in acantotic tissue a decrease in scattering and an increase in the probing depth of the laser beam, and, therefore, an increase in the diagnostic volume.

Thus, the observed increases in the parameters of the microcirculation of the psoriatic plaque may be due to a combination of morphological (dilatation of vessels - increase in their number, width and length), dynamic (increased blood flow), and optical effects (partial enlightenment of the near-surface layers of the skin due to acanthosis). 


\section{CONCLUSION}

Microcirculatory disorders are a characteristic feature of psoriasis and play a decisive role in its pathogenesis. Capillary loops in psoriatic plaques are elongated, dilated and tortuous.

The conducted studies showed that the LDF method can not only control the degree of change in the intensity of the microcirculation of blood, but also analyse the state of the functional mechanisms of controlling the microcirculation directly in the plaque and intact skin.

The obtained data confirm the earlier studies that the blood flow measured in the plaque is much higher than that recorded in the intact region. In this case, the dominance of all types of oscillations in psoriatic lesions is revealed in comparison with the zone not involved in the pathological process. Monitoring the intensity of blood flow in psoriatic plaques can provide an opportunity to determine the degree of "activity" of psoriasis, monitor the effectiveness of the therapy and perform individual selection of medicines. Evaluation of the contribution of each link of the microvascular bed, taking part in the modulation of blood flow, can contribute to further study of the pathophysiology of the disease.

\section{ACKNOWLEDGMENTS}

This work was supported by the Russian foundation for basic research RFBR 17-41-590560. The authors also acknowledge the support of a grant of the President of the Russian Federation for state support of young Russian scientists № MK-3400.2018.8. Evgeny Zherebtsov kindly acknowledges funding from the European Union’s Horizon 2020 research and innovation programme under the Marie Sklodowska-Curie grant agreement No. 703145.

\section{REFERENCES}

[1] Greb, J. E., Goldminz, A. M., Elder, J. T., Lebwohl, M. G., Gladman, D. D., Wu, J. J., Mehta, N. N., Finlay, A. Y., Gottlieb, A. B., "Psoriasis.," Nat. Rev. Dis. Prim. 2, 16082 (2016).

[2] De Angelis, R., Bugatti, L., Del Medico, P., Nicolini, M.., Filosa, G., "Videocapillaroscopic Findings in the Microcirculation of the Psoriatic Plaque," Dermatology 204(3), 236-239 (2002).

[3] Micali, G., Lacarrubba, F., Musumeci, M. L., Massimino, D.., Nasca, M. R., "Cutaneous vascular patterns in psoriasis.," Int. J. Dermatol. 49(3), 249-256 (2010).

[4] Zherebtsov, E. A., Zherebtsova, A. I., Doronin, A., Dunaev, A. V., Podmasteryev, K. V., Bykov, A.., Meglinski, I., "Combined use of laser Doppler flowmetry and skin thermometry for functional diagnostics of intradermal finger vessels," J. Biomed. Opt. 22(4), 40502 (2017).

[5] Mizeva, I., Makovik, I., Dunaev, A., Krupatkin, A.., Meglinski, I., "Analysis of skin blood microflow oscillations in patients with rheumatic diseases.,” J. Biomed. Opt. 22(7), 70501 (2017).

[6] Makovik, I. N., Dunaev, A. V., Dremin, V. V., Krupatkin, A. I., Sidorov, V. V., Khakhicheva, L. S., Muradyan, V. F., Pilipenko, O. V., Rafailov, I. E., et al., "Detection of angiospastic disorders in the microcirculatory bed using laser diagnostics technologies," J. Innov. Opt. Health Sci. 11(1) (2018).

[7] Dremin, V. V., Zherebtsov, E. A., Sidorov, V. V., Krupatkin, A. I., Makovik, I. N., Zherebtsova, A. I., Zharkikh, E. V., Potapova, E. V., Dunaev, A. V., et al., "Multimodal optical measurement for study of lower limb tissue viability in patients with diabetes mellitus," J. Biomed. Opt. 22(8) (2017).

[8] Santesson, P., Lins, P.-E., Kalani, M., Adamson, U., Lelic, I., von Wendt, G., Fagrell, B.., Jorneskog, G., "Skin microvascular function in patients with type 1 diabetes: An observational study from the onset of diabetes.," Diabetes Vasc. Dis. Res. 14(3), 191-199 (2017).

[9] Dunaev, A. V., Sidorov, V. V., Krupatkin, A. I., Rafailov, I. E., Palmer, S. G., Stewart, N. A., Sokolovski, S. G.., Rafailov, E. U., "Investigating tissue respiration and skin microhaemocirculation under adaptive changes and the synchronization of blood flow and oxygen saturation rhythms.," Physiol. Meas. 35(4), 607-621 (2014).

[10] Dunaev, A. V., Dremin, V. V., Zherebtsov, E. A., Rafailov, I. E., Litvinova, K. S., Palmer, S. G., Stewart, N. A., Sokolovski, S. G.., Rafailov, E. U., "Individual variability analysis of fluorescence parameters measured in skin 
with different levels of nutritive blood flow," Med Eng Phys 37(6), 574-583 (2015).

[11] Tankanag, A., Chemeris, N., "Application of the adaptive wavelet transform for analysis of blood flow oscillations in the human skin.," Phys. Med. Biol. 53(21), 5967-5976 (2008).

[12] Hendriks, A. G. M., Steenbergen, W., Hondebrink, E., van Hespen, J. C. G., van de Kerkhof, P. C. M.., Seyger, M. M. B., "Whole field laser Doppler imaging of the microcirculation in psoriasis and clinically unaffected skin," J. Dermatolog. Treat. 25(1), 18-21 (2014).

[13] Hern, S., Stanton, A. W. B., Mellor, R. H., Harland, C. C., Levick, J. R.., Mortimer, P. S., "Blood flow in psoriatic plaques before and after selective treatment of the superficial capillaries," Br. J. Dermatol. 152(1), 6065 (2005).

[14] Auer, T., Bacharach-Buhles, M., el-Gammal, S., Stucker, M., Panz, B., Popp, C., Hoffmann, K., Happe, M., Altmeyer, P., "The hyperperfusion of the psoriatic plaque correlates histologically with dilatation of vessels.," Acta Derm. Venereol. Suppl. (Stockh). 186, 30-32 (1994). 